\title{
A!
}

This is an electronic reprint of the original article.

This reprint may differ from the original in pagination and typographic detail.

Jena, P.; Manninen, Matti; Nieminen, R. M.; Puska, M. J.

\section{Muon states in uniaxially strained iron}

Published in:

Physical Review B

DOI:

10.1103/PhysRevB.29.4170

Published: 01/04/1984

Document Version

Publisher's PDF, also known as Version of record

Please cite the original version:

Jena, P., Manninen, M., Nieminen, R. M., \& Puska, M. J. (1984). Muon states in uniaxially strained iron. Physical Review B, 29(7), 4170-4172. https://doi.org/10.1103/PhysRevB.29.4170

This material is protected by copyright and other intellectual property rights, and duplication or sale of all or part of any of the repository collections is not permitted, except that material may be duplicated by you for your research use or educational purposes in electronic or print form. You must obtain permission for any other use. Electronic or print copies may not be offered, whether for sale or otherwise to anyone who is not an authorised user. 


\title{
Muon states in uniaxially strained iron
}

\author{
P. Jena \\ Physics Department, Virginia Commonwealth University, Richmond, Virginia 23284 \\ M. Manninen \\ Research Institute for Theoretical Physics, University of Helsinki, \\ SF-00170 Helsinki, Finland \\ R. M. Nieminen \\ Department of Physics, University of Jyväskylä, SF-40720 Jyväskylä, Finland \\ M. J. Puska \\ Laboratory of Physics, Helsinki University of Technology, SF-23150 Espoo 15, Finland
}

(Received 17 October 1983)

\begin{abstract}
Effects of lattice relaxation, quantum motion, and uniaxial strain on the internal field at a positive-muon site in iron have been calculated. The uniaxial strain gives rise to a statistical shift of the muon population at interstitial sites. The effect of the population shift is found to be primarily responsible for the observed changes in the muon-precession frequency. The theory also predicts a $1 / T$ temperature dependence of the frequency shifts.
\end{abstract}

In this Rapid Communication we report the first $a b$ initio calculation of the energetics of a positive muon $\mu^{+}$in uniaxially strained iron. Using a self-consistent procedure based on the effective-medium theory ${ }^{1,2}$ we show that the otherwise degenerate energy states of an interstitial muon are split when stress is applied along the $\langle 100\rangle$ direction which is also the axis of magnetization. This splitting induces a statistical population shift between magnetically inequivalent interstitial sites. Consequently, the dipolar interaction between the muon and iron spins gives rise to a shift in the muon-precession frequency. Other interactions of the muon spin with the environment have been found to be negligible. The calculated change in the muon internal field $\partial B_{\mu}$ agrees well with experiment both in magnitude and sign. We also find $\partial B_{\mu}$ to be strongly temperature dependent. For $T>77 \mathrm{~K}, \partial B_{\mu} \propto M_{s}(T) 1 / T$ to within an accuracy of $1 \%$, where $M_{s}(T)$ is the temperature-dependent host magnetization. Our theory takes into account the distortions of host atoms due to $\mu^{+}$self-consistently. The effect of the muon zero-point motion on the average dipolar field is included in the calculation and found to be large.

The use of muons as microscopic probes of magnetic fields in ferromagnetic metals is well known. ${ }^{3}$ The internal field experienced by the $\mu^{+}$is given by

$$
\overrightarrow{\mathrm{B}}_{\mu}=\overrightarrow{\mathrm{B}}_{\mathrm{ext}}+\overrightarrow{\mathrm{B}}_{\mathrm{dem}}+\overrightarrow{\mathrm{B}}_{L}+\overrightarrow{\mathrm{B}}_{\mathrm{dip}}+\overrightarrow{\mathrm{B}}_{\mathrm{hf}}
$$

where $B_{\text {ext }}$ and $B_{\text {dem }}$ are, respectively, the external and demagnetizing field. The Lorentz field $B_{L}$ is expressed in terms of the saturation magnetization density $M_{s}$ :

$$
\overrightarrow{\mathrm{B}}_{L}=\frac{4 \pi}{3} \overrightarrow{\mathrm{M}}_{s}
$$

The dipole field $B_{\text {dip }}$ arising from the interaction of the muon magnetic moment $\mu_{0}$ with the magnetic moment $\mu_{\text {ion }}$ of $\mathrm{Fe}$ ions a distance $r_{j}$ from the muon has the form

$$
\overrightarrow{\mathrm{B}}_{\mathrm{dip}}=\frac{\mu_{0}}{4 \pi} \sum_{j} \frac{3 \overrightarrow{\mathrm{r}}_{j}\left(\overrightarrow{\mathrm{r}}_{j} \cdot \vec{\mu}_{\text {ion }}\right)-r_{j}^{2} \vec{\mu}_{\text {ion }}}{r_{j}^{5}} .
$$

$B_{\mathrm{hf}}$ is the hyperfine field due to the Fermi contact interaction of the muon spin with the spin-polarized conduction electrons.

In bcc iron all the interstitial sites (tetrahedral and octahedral) have tetragonal symmetry. While the interstitial tetrahedral (octahedral) sites are structurally equivalent, magnetically there are two different tetrahedral (octahedral) sites. The dipolar fields experienced by various interstitial muon sites are shown in Fig. 1. There are twice as many tetrahedral sites with $B_{\text {dip }}=+2.6 \mathrm{kG}$ as those with $B_{\text {dip }}=-5.2 \mathrm{kG}$. Similarly, the octahedral sites with $B_{\text {dip }}=-9.25 \mathrm{kG}$ are twice as many as those with $B_{\text {dip }}=+18.5 \mathrm{kG}$. Thus one would normally expect a $\mu^{+}$to precess with two distinctly different frequencies. The fact that only a single muon-precession frequency was observed in perfect $\mathrm{Fe}$ implied that the muon diffuses rapidly between the interstitial sites, thus averaging the dipole field

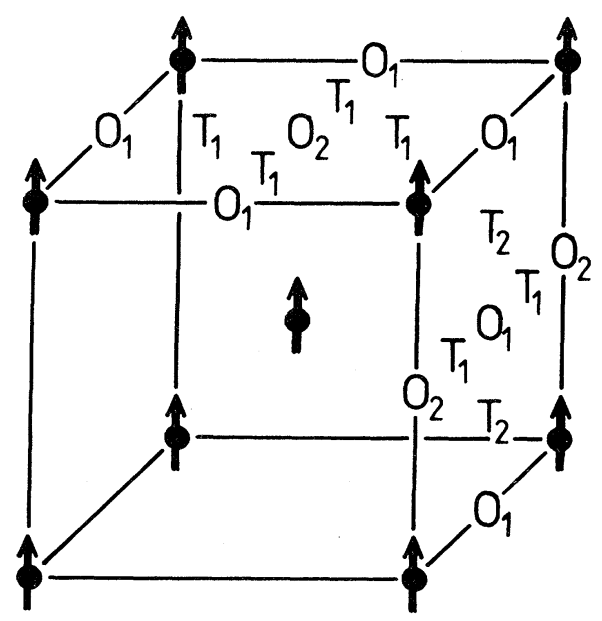
sites. 
to zero. The hyperfine field extracted from the measured muon internal field $B_{\mu}$ in Eq. (1) is now well understood theoretically. ${ }^{4}$

The change in $B_{\mu}$ in Eq. (1) due to the application of uniaxial strain $\epsilon$ is given by

$$
\frac{\partial B_{\mu}}{\partial \epsilon}=\frac{\partial B_{L}}{\partial \epsilon}+\frac{\partial B_{\mathrm{dip}}}{\partial \epsilon}+\frac{\partial B_{\mathrm{hf}}}{\partial \epsilon} .
$$

By applying stress along $\langle 100\rangle$ direction, Namkung et al. ${ }^{5,6}$ have observed a frequency shift of $-0.34 \pm 0.023$ $\mathrm{MHz}$ per 100 microstrain. This corresponds to $\partial B_{\mu} / \partial \epsilon$ $=+25.1 \pm 1.7 \mathrm{G} / 100$ microstrain. We estimate the change in the Lorentz field $B_{L}$ from measured change in magnetization under pressure and compressibility to be $+0.3 \mathrm{G}$ per 100 microstrain. The change in the hyperfine field is found to be about $-1 \mathrm{G}$ per 100 microstrain from the pressuredependence study of Hartmann and co-workers. ${ }^{7}$ The change in the dipole field due to the new positions of iron atoms under strain is again of the order of $1 \mathrm{G}$. Our estimates are in agreement with those of Namkung et al. ${ }^{5,6}$

The above analysis clearly indicated that the origin of the observed frequency shift has to lie elsewhere. Namkung et al. asserted that the uniaxial stressd can cause a population shift of the muons by lifting the degeneracy of the interstitial sites. Using the earlier results of Sugimoto and Fukai $^{8}$ on muon states in $\mathrm{Nb}$ and $\mathrm{V}$, these authors were able to get an order-of-magnitude agreement with experiment. In the following we confirm this assertion by means of a first-principles self-consistent calculation of muon states in strained iron.

We begin by summarizing the salient features of our calculation. Following Sugimoto and Fukai ${ }^{8}$ we write the total energy of the muon-metal system in the adiabatic limit as

$$
E(\{\overrightarrow{\mathrm{u}}(\overrightarrow{\mathrm{R}})\}, \mu)=E_{L}(\{\overrightarrow{\mathrm{u}}(\overrightarrow{\mathrm{R}})\})+E_{\mu}(\{\overrightarrow{\mathrm{u}}(\overrightarrow{\mathrm{R}})\}),
$$

where $\{\overrightarrow{\mathbf{u}}(\overrightarrow{\mathrm{R}})\}$ is the set of host atom displacements, $E_{L}$ the energy stored in the strained lattice, and $E_{\mu}$ the muon energy eigenvalue. Minimizing Eq. (5) with respect to the muon degree of freedom leads to the muon Schrödinger equation

$$
\left(-\frac{\hbar^{2}}{2 m_{\mu}} \nabla^{2}+V(\vec{r})\right) \psi_{\mu}(\vec{r})=E_{\mu} \psi_{\mu}(\vec{r}) .
$$

$V(\vec{r})$ is the potential-energy field sensed by the muon. It arises from the "embedding" interaction between the impurity quasiatom (muon) and the host. The construction of $V(\vec{r})$ in Eq. (6) is discussed below. In the harmonic approximation, the lattice energy $E_{L}$ is expressible in terms of the dynamic matrix of the solid. Following the Kanzaki formalism, we can evaluate the atomic displacements $u$ (and the elastic energy $E_{L_{1}}$ ) in terms of the lattice Green's function $G$, if the forces on the atoms are known. In matrix shorthand notation,

$$
\overrightarrow{\mathrm{u}}=\underline{G} \overrightarrow{\mathrm{F}}^{*} \text {, }
$$

where $\overrightarrow{\mathrm{F}}^{*}$ is the Kanzaki force on an atom in a relaxed position $\vec{R}+\vec{u}(\vec{R})$ :

$\overrightarrow{\mathrm{F}}^{*}(R)=-\int d^{3} r\left|\psi_{\mu}(\overrightarrow{\mathrm{r}})\right|^{2} \frac{\partial V(|\overrightarrow{\mathrm{R}}+\overrightarrow{\mathrm{u}}(\overrightarrow{\mathrm{R}})-\overrightarrow{\mathrm{r}}|)}{\partial[|\overrightarrow{\mathrm{R}}+\overrightarrow{\mathrm{u}}(\overrightarrow{\mathrm{r}})-\overrightarrow{\mathrm{r}}|]}$.

For a delocalized muon the force and the displacements vanish, but if $\psi_{\mu}(\vec{r})$ is localized in space, the self- consistent iteration of Eqs. (2) - (4) may lead to overall energy lowering in Eq. (5). This corresponds to self-trapping in an otherwise ideal crystal. Technically, the muon Schrödinger equation (6) is solved numerically with use of three-dimensional relaxation techniques, ${ }^{9}$ using appropriate boundary conditions. The lattice Green's function, which is only a property of the host, is evaluated using detailed discrete lattice phonon data. ${ }^{10}$

Sugimoto and Fukai $^{8}$ constructed $V(\vec{r})$ by a pairwise summation utilizing an empirical muon (hydrogen)-metal central pair potential. Instead, we formulate the interaction energy surfaces $V(\vec{r})$ along the lines introduced by Stott and Zaremba ${ }^{2}$ and Nørskov and Lang. ${ }^{1}$ First, we assume that a satisfactory approximation for the electron density $n(\vec{r})$ in the host (without the muon, but with atomic relaxation and/or defects) is available. For most purposes, a superposition of neutral atom electron densities is accurate enough. The electronic perturbation due to the impurity is, due to metallic screening, well localized in space, say within a sphere of radius $R_{a}$. Inside this sphere the charge density $\rho_{a}(\vec{r})$ and the Coulomb potential $\phi_{a}(\vec{r})$ of the impurity quasiatom are taken to be equal to those for a hydrogen atom embedded in a homogeneous electron gas. ${ }^{11}$ The density $\bar{n}_{0}$ where the embedding is assumed to take place is chosen by using $\phi_{a}(\vec{r})$ as the sampling function for the unperturbed host:

$$
\bar{n}_{0}=\int_{a} d^{3} r \phi_{a}(\overrightarrow{\mathrm{r}}) n_{0}(\overrightarrow{\mathrm{r}}) / \int_{a} d^{3} r \phi_{a}(\overrightarrow{\mathrm{r}}) .
$$

We then proceed systematically in perturbation theory and obtain (for more details, see Ref. 12)

$$
V(\overrightarrow{\mathrm{r}})=\Delta E^{\mathrm{hom}}\left(\bar{n}_{0}(\overrightarrow{\mathrm{r}})\right)-\alpha \bar{n}_{0}(\overrightarrow{\mathrm{r}})+E_{\mathrm{hyb}},
$$

where $\Delta E^{\text {hom }}$ is the immersion energy of the muon in the homogeneous electron gas of the sampled density $\bar{n}_{0}, \alpha$ is a constant arising from the first-order (Coulomb) correction, and $E_{\text {hyb }}$ is the second-order (hybridization) term. This procedure has been applied successfully for calculating hydrogen heats of solution ${ }^{12}$ and chemisorption energies of hydrogen on transition metals. ${ }^{13}$

The self-consistent solution of Eqs. (6)-(10) gives the lattice relaxation around the impurity and the self-trapping energy, i.e., the difference $(<0)$ between the self-trapped and delocalized-state eigenvalues plus the energy $(>0)$ used in the lattice deformation. We do not try to estimate the muon diffusion rate in a unstrained lattice in this Rapid Communication. In Ref. 9 possible diffusion mechanisms are discussed on the basis of the results of the present calculation scheme. Results for the iterstitial muon in iron are given in Table I. The octahedral site seems to be energetically favorable. Both the lattice relaxation and the muon zero-point motion affect the average dipolar field seen by the muon. The lattice relaxation tends to increase the symmetry of the interstitial site and decrease the dipolar field. In the octahedral site the muon distribution extends towards the neighboring tetrahedral sites, as shown in Fig. 2. Thus the average field

$$
B_{\text {dip }}^{\text {av }}=\int d^{3} r\left|\psi_{\mu}(\overrightarrow{\mathrm{r}})\right|^{2} B_{\text {dip }}(\overrightarrow{\mathrm{r}})
$$

is smaller than the field at the center of the octahedral site. In the tetrahedral site the effect of the zero-point motion is smaller. The results are given in Table I. Note that the average field over all octahedral (tetrahedral) sites remains equal to zero. 
TABLE I. Results for $\mu^{+}$in iron. $\delta R_{1} / R_{1}$ and $\delta R_{2} / R_{2}$ are changes in the nearest- and second-nearest-neighbor distances. $E_{\mathrm{st}}$ is the self-trapping energy relative to the delocalized band state, the energy eigenvalue of which is $0.66 \mathrm{eV}$ above the (underformed) potential minimum and $0.60 \mathrm{eV}$ below the energy of free muonium in vacuum. $B_{\text {dip }}^{0}$ and $B_{\text {dip }}$ are the dipolar fields in the center of the interstitial site without and with the lattice relaxation due to the muon. $B_{\text {dip }}^{\text {av }}$ is the average field over the muon distribution. The values for the magnetically inequivalent site are given in parentheses. $\Delta E$ is the energy difference between the two inequivalent sites in strained iron (100 microstrain). $\left\langle B_{\text {dip }}^{\text {av }}\right\rangle$ is the thermal average of the dipolar field at $300 \mathrm{~K}$ in strained iron (100 microstrain).

\begin{tabular}{|c|c|c|}
\hline & $\begin{array}{l}\text { Octahedral } \\
\text { site }\end{array}$ & $\begin{array}{c}\text { Tetrahedra } \\
\text { site }\end{array}$ \\
\hline$\delta R_{1} / R_{1}(\%)$ & 10.6 & 6.0 \\
\hline$\delta R_{2} / R_{2}(\%)$ & 0.5 & -0.3 \\
\hline$E_{\mathrm{st}}(\mathrm{eV})$ & -0.36 & -0.33 \\
\hline$B_{\text {dip }}^{0}(\mathrm{kG})$ & $-9.25(18.5)$ & $2.6(-5.2)$ \\
\hline$B_{\text {dip }}(\mathrm{kG})$ & $-5.6 \quad(11.2)$ & $2.2(-4.4)$ \\
\hline$B_{\text {dip }}^{\text {av }}(\mathrm{kG})$ & $-3.8 \quad(7.6)$ & $2.2(-4.4)$ \\
\hline$\Delta E(\mathrm{meV})$ & -0.19 & 0.53 \\
\hline$\left\langle B_{\text {dip }}^{\text {av }}\right\rangle(G)$ & 18 & 29 \\
\hline
\end{tabular}

A uniaxial strain makes the different octahedral (tetrahedral) sites inequivalent. The average field due to the population difference at a finite temperature is

$$
\left\langle B_{\text {dip }}^{\text {av }}\right\rangle=\frac{2 B_{\text {dip }_{1}}^{\text {av }}(T) e^{-E_{1} / k T}+B_{\text {dip }_{2}}^{\text {av }}(T) e^{-E_{2} / k T}}{e^{-E_{2} / k T}+2 e^{-E_{1} / k T}},
$$

where the subscripts 1 and 2 refer to the two inequivalent octahedral (tetrahedral) interstitial sites. Assuming that

$$
B_{\text {dip }}(T)=B_{\text {dip }}(0) M_{s}(T) / M_{s}(0),
$$

and that $\Delta E \equiv\left(E_{1}-E_{2}\right)<<k T$ one can write

$$
B_{\text {dip }}^{\text {av }}=\frac{2}{3} B_{\text {dip }_{1}}^{\text {av }(0)} \frac{M_{s}(T)}{M_{s}(0)} \frac{\Delta E}{k T} .
$$

We have calculated $\Delta E$ for various strengths of strain up to $5 \times 10^{4}$ microstrain and found it to be linear in strain. The lattice Green's function was assumed to be unaffected by the strain. The results for 100 microstrain are shown in Table I. The average dipolar field in strained iron at room

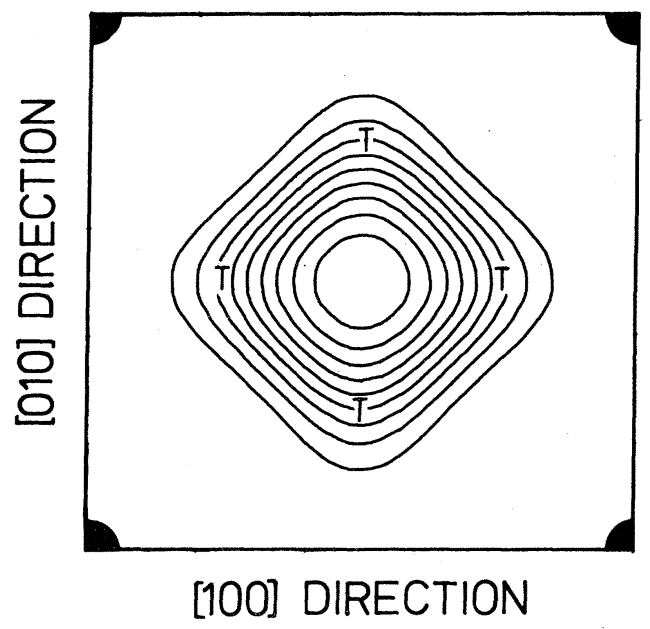

FIG. 2. Muon distribution at interstitial sites.

temperature $(300 \mathrm{~K})$ agrees well with the experimental result of $25.1 \pm 1.7 \mathrm{G} / 100$ microstrain.

In calculating the dipolar field the change in the atomic positions due to the strain was neglected. Actually, the atomic displacements make the average field over different interstitial sites nonzero, $-7.1 \mathrm{G} / 100$ microstrain for the octahedral site, and $-2.3 \mathrm{G} / 100$ microstrain for the tetrahedral site. However, these numbers will be reduced as a result of the lattice relaxation around the muon and also the muon zero-point motion to about $-2 \mathrm{G} / 100$ microstrain and $-1 \mathrm{G} / 100$ microstrain, respectively.

In conclusion, we have made a first-principles calculation of the effects of lattice relaxation, zero-point motion, and uniaxial strain on the average dipolar field seen by a muon in iron. The results indicate that in magnetic materials where the muon is localized, the lattice relaxation and muon zero-point motion will significantly change the dipolar field and thus the muon-precession frequency. Calculation of the average dipolar field in uniaxially strained iron is in good agreement with the experimental result at room temperature. In order to establish the origin of the muon frequency shift under strain unambiguously, experiments should be performed at different temperatures and for strain along other directions. It is hoped that such experiments will soon be carried out.

The authors would like to thank W. J. Kossler, P. F. Meier, and J. K. Nørskov for useful discussions.
1 J. K. Nørskov and N. D. Lang, Phys. Rev. B 21, 2131 (1980).

${ }^{2}$ M. J. Stott and E. Zaremba, Phys. Rev. B 22, 1564 (1980).

${ }^{3}$ A. B. Denison, H. Graf, W. Kundig, and P. F. Meier, Helv. Phys. Acta 52, 460 (1979).

${ }^{4}$ B. Lindgren and D. E. Ellis, Phys. Rev. B 26, 636 (1982).

${ }^{5}$ M. Namkung, W. J. Kossler, C. E. Stronach, R. I. Grynszpan, and B. D. Patterson, in Proceedings of the Yamada Conference on Muon Spin Rotation, Shimoda, Japan, 1983 (unpublished).

${ }^{6} \mathrm{M}$. Namkung, Ph.D. thesis, College of William and Mary, Virginia, 1982 (unpublished).
${ }^{7}$ T. Bute, J. Chappert, J. F. Dufresne, O. Hartman, E. Karlsson, B. Lindgren, L. O. Norlin, P. Pondini, and A. Yaouanc, Phys. Lett. 75A, 321 (1980).

${ }^{8}$ H. Sugimoto and Y. Fukai, Phys. Rev. B 22, 670 (1980).

${ }^{9}$ M. J. Puska and R. M. Nieminen (unpublished).

${ }^{10}$ I. R. MacGillivray and C. A. Sholl, J. Phys. F 13, 23 (1983).

${ }^{11}$ M. J. Puska, R. M. Nieminen, and M. Manninen, Phys. Rev. B 24, 3037 (1981).

12J. K. Nørskov, Phys. Rev. B 26, 2875 (1982).

${ }^{13}$ P. Norlander, S. Hollaway, and J. K. Nørskov (unpublished). 